\title{
IncRNA 430945 promotes the proliferation and migration of vascular smooth muscle cells via the ROR2/RhoA signaling pathway in atherosclerosis
}

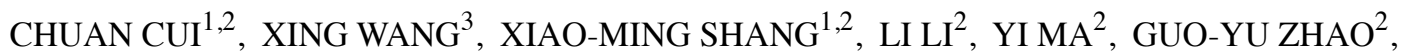 \\ YU-XIN SONG ${ }^{2}$, XUE-BIN GENG $^{2}$, BI-QIONG ZHAO ${ }^{2}$, MEI-RONG TIAN ${ }^{2}$ and HONG-LING WANG ${ }^{2}$ \\ ${ }^{1}$ Department of Internal Medicine, Hebei Medical University, Shijiazhuang, Hebei 050011; \\ ${ }^{2}$ Department of Cardiology, Tangshan Workers Hospital, Tangshan, Hebei 063000; ${ }^{3}$ Department of Internal Medicine, \\ Qian'an Hospital of Traditional Chinese Medicine, Qian'an, Hebei 064400, P.R. China
}

Received August 15, 2018; Accepted March 27, 2019

DOI: $10.3892 / \mathrm{mmr} .2019 .10137$

\begin{abstract}
The proliferation and migration of vascular smooth muscle cells (VSMCs) are major cellular events in hypertension-induced vascular remodeling, which is closely involved in the progression of atherosclerosis (AS). Although long non-coding RNAs (lncRNAs) are gaining recognition as novel regulators of VSMCs, their functioning and role in AS remain to be elucidated. In the present study, the role of lncRNA ENST00000430945 (lncRNA 430945) in AS was investigated. VSMCs transfected with a small interfering RNA (siRNA; si-430945) and a negative control (si-NC) were used. Cell Counting Kit- 8 , wound-healing and Transwell migration arrays were performed to determine whether IncRNA 430945 influenced VSMC proliferation and migration. Furthermore, the study examined whether a correlation exists between lncRNA 430945 and the receptor tyrosine kinase-like orphan receptor 2 (ROR2) signaling pathway. It was found that the expression of IncRNA 430945 was high in human AS tissues, which in turn promoted angiotensin II (AngII)-induced VSMC proliferation. Reverse transcription-quantitative polymerase chain reaction (RT-qPCR) and western blot analyses showed that IncRNA 430945 mediated the AngII-induced upregulation of ROR2. In addition, the microarray and RT-qPCR results showed that the expression of IncRNA 430945 was increased considerably in AS tissues. The downregulation of IncRNA 430945 significantly suppressed AngII-induced VSMC proliferation and migration. In addition, ROR2 levels in VSMCs transfected with si-430945 were markedly lower than those cells transfected with si-NC. Additionally, western
\end{abstract}

Correspondence to: Dr Xiao-Ming Shang, Department of Internal Medicine, Hebei Medical University, 361 Zhongshan East Road, Shijiazhuang, Hebei 050011, P.R. China

E-mail: shxm@vip.163.com

Key words: vascular smooth muscle cells, long non-coding RNA, atherosclerosis, signaling pathway, angiotensin II blotting showed that IncRNA 430945 activated the signaling pathways associated with ROR2 and Ras homolog gene family member A (RhoA). The upregulation of lncRNA 430945 in AS promoted the proliferation and migration of VSMCs via activation of the ROR2/RhoA signaling pathway. Therefore, targeting ROR2 or RhoA may be a promising technique in developing therapeutic strategies for treating AS.

\section{Introduction}

Atherosclerosis (AS) is one of the leading causes of mortality in the world. It is a multistep cardiovascular disease promoted by several risk factors, including the accumulation of macrophages, production of pro-inflammatory cytokines and dysfunction of endothelial and vascular smooth muscle cells (VSMCs) (1). Vascular remodeling is closely linked to the progression of AS, and the proliferation and migration of VSMCs is a major cellular event in hypertension- and diabetes-induced vascular remodeling (2). However, the detailed mechanism underlying VSMC proliferation and migration in AS remains to be fully elucidated.

Long non-coding RNAs (lncRNAs) are RNA molecules present in eukaryotic cells, which are $>200$ nucleotides long and are not translated into protein. IncRNAs are involved in several physiological and pathological processes, including carcinogenesis (3) and cardiovascular diseases (4). Recent evidence shows that IncRNAs are key in the development of AS by regulating gene expression at the transcriptional and post-transcriptional levels, which in turn influences multiple signaling pathways with respect to the proliferation and migration of VSMCs (5-7). This indicates that targeting lncRNAs in treating AS may be promising; however, the function of lncRNAs and the underlying mechanism by which they influence the proliferation and migration of VSMCs remain to be fully elucidated.

Receptor tyrosine kinase-like orphan receptor 2 (ROR2) is a member of the tyrosine kinase receptor family, which acts as a receptor of Wnt5a (8). Wnt5a/ROR2 signaling primarily activates $\beta$-catenin-independent non-canonical Wnt pathways, which involve various signal mediators, 
including c-Jun N-terminal kinase, filamin A, Rous sarcoma oncogene (c-Src) and resistance to audiogenic seizures (Ras) homolog gene family member A (RhoA), thereby regulating cell proliferation and motility $(9,10)$. The sustained overactivation of RhoA is widely implicated in the pathogenesis of several cardiovascular disorders, including hypertension (11). Previous studies have examined the regulation of RhoA by non-canonical Wnt signals and the transduction of non-canonical Wnt signaling by small Rho GTPases (12), and increasing evidence supports to the role of lncRNAs in AS (13). lncRNA ENST00000430945 (lncRNA 430945) is a large, intergenic non-coding RNA located on human chromosome 9q22.31. Microarray analysis and lncRNA-mRNA co-expression profiling has showed that lnc43094 is closely linked to the ROR2 signaling pathway. However, the specifics of lncRNA 430945-based ROR2 modulation in AS remain to be elucidated.

The present study investigated the role of lncRNAs and the putative molecular mechanisms by which they regulate VSMC proliferation and migration in AS. The expression of lncRNA 430945 was detected in human AS tissues and VSMCs, in addition to examining the proliferation and migration of angiotensin II (AngII)-induced VSMCs. The final aim was to elucidate the molecular mechanisms by which lncRNA 430945 regulates the expression of ROR 2 and the proliferation and migration of VSMCs.

\section{Materials and methods}

Reagents. Dulbecco's modified Eagle's medium (DMEM, Hyclone; GE Healthcare Life Sciences, Logan, UT, USA), fetal bovine serum (FBS, Gibco; Thermo Fisher Scientific, Waltham, MA, USA) and penicillin and streptomycin were purchased from Gibco (Thermo Fisher Scientific, Waltham, MA, USA). Anti- $\beta$-actin (cat. no. 60008-1-Ig), anti-RhoA (cat. no. 10749-1-AP), anti-proliferating cell nuclear antigen (PCNA; cat. no. 10205-2-AP), anti-matrix metallopeptidase 9 (MMP9; cat. no. 10375-2-AP) and anti-ROR2 (cat. no. 10616-1-AP) antibodies were purchased from ProteinTech Group, Inc., (Chicago, IL, USA). The lncRNA430945 expression vector, pcDNA-lncRNA430945, was synthetized and constructed by Guangzhou Ribobio Co., Ltd., (Guangzhou, China). Small interfering (si)RNA-lncRNA 430945 (si-lncRNA430945) and siRNA-ROR2 (si-ROR2) were obtained from Merck KGaA (Darmstadt, Germany). Cells were transfected with pcDNA-lncRNA 430945 or siRNAs using Lipofectamine ${ }^{\circledR} 2000$ (Invitrogen; Carlsbad, CA, USA) following the manufacturer's protocol.

Human tissue harvest. The AS tissue specimens were obtained in the operating room from three male patients undergoing elective open aortic aneurysm repair. The mean age of the patients was 52 years (range, 47-63 years). Normal aortic wall tissue specimens were obtained from age-matched organ donors to serve as controls $(n=3)$. All tissue specimens were collected at the Department of Cardiology, Tangshan Workers Hospital (Tangshan, China) between May 2017 and January 2018. All patients and healthy volunteers were well informed. One section of each aortic wall specimen was fixed overnight in $10 \%$ neutral-buffered formalin and processed for routine embedding in paraffin. An adjacent tissue region was snap-frozen in liquid nitrogen, stored at $-80^{\circ} \mathrm{C}$, and subsequently used for protein and nucleic acid extraction. The procedure of the experiment was approved by the Institutional Review Board of Hebei Medical University (Shijiazhuang, China) and written informed consent was obtained from all patients.

Microarray analysis. Total RNA was extracted from the three pairs of aortic tissue samples from the patients with AS and controls. Total RNA was extracted and RNA quality was determined. The Quick Amp Labeling kit, One-Color (cat. no. 5190-2305; Agilent Technologies, Inc., Santa Clara, CA, USA) was used to carry out the labeling reaction, following which the labeled/amplified RNA was purified and the labeled cRNA quality was determined. Hybridization was performed using an Agilent Gene Expression Hybridization kit (cat. no. 5188-5242; Agilent Technologies, Inc.) according to the manufacturer's protocol and the microarray was washed. An Agilent microarray scanner (model G2565AA; Agilent Technologies, Inc.) was used and Agilent Feature Extraction software v.11.0.1.1 (Agilent Technologies, Inc.) was used to extract data.

The microarray was performed by KangChen Biotech (Zhejiang Kangchen Biotech Co., Ltd., Wuhan, China). By comparing the normalized expression levels in the AS and non-AS serum samples, differentially expressed lncRNAs with statistical significance $(\mathrm{A} / \mathrm{N}$ or $\mathrm{N} / \mathrm{A}$ fold change $>1.5$, $\mathrm{P}<0.05)$ were identified with a paired t test; A refers to AS and $\mathrm{N}$ refers to Normal).

Mouse model of carotid artery ligation. The carotid artery ligation (CAL) was performed according to the protocol developed by Allagnat et al (14), on 8-week-old male C57BL/6J mice, as previously described. Mice were fed standard laboratory chow and water ad libitum, and were housed in single cages under specific pathogen-free conditions (temperature and humidity were controlled at $22 \pm 2^{\circ} \mathrm{C}$ and $55 \pm 5 \%$ ) in a room with a 12-h light/dark cycle. Male mice (weight, 20-25 g; age, 8 weeks) were used in the present study. Briefly, the mice were anesthetized with $4 \%$ chloral hydrate. The left common carotid artery was dissected through a small cervicotomy and ligated with prolene 7.0 just below the carotid bifurcation. The mice were sacrificed by cervical dislocation, and perfused with PBS followed by buffered paraformaldehyde $4 \%$ through the left ventricle prior to CAL for reference or on days 3, 14 or 28 days following CAL. All animal experimentation conformed to the Guidelines of the local Animal Care and Use Committee at Tangshan Workers Hospital.

Cell culture. Mouse vascular smooth muscle cells (VSMCs) were purchased from the Institute of Biochemistry Cell Biology (Shanghai, China). The cells were cultured at $37^{\circ} \mathrm{C}$ in a humidified incubator with $5 \% \mathrm{CO}_{2}$ and $95 \%$ air, and maintained in DMEM supplemented with $10 \%$ FBS and $1 \%$ penicillin/streptomycin $(100 \mathrm{U} / \mathrm{ml} / 100 \mathrm{mg} / \mathrm{ml})$.

Cell transfection. The VSMCs were seeded into the 6-well plates at a density of 5,000 cells/well, cultured for $24 \mathrm{~h}$ and then transfected when the cell density reached $70 \%$. The siRNA sequences of IncRNA 430945 and ROR2 were 
as follows: si-lncRNA 430945: 5'-ACUCCUUCGUAU CUCGCCTT-3', si-ROR2: 5'-AGUCUCUCAUAUCUUCGG T-3' and a negative control (a scrambled matched \% GC oligonucleotide). Following mixing of Lipofectamine ${ }^{\circledR} 2000$ with the pcDNA-lncRNA430945, pc-DNA, si-lncRNA430945, si-ROR2 or the negative transfection group (si-NC), and the mixture was added to the cells and incubated for $6 \mathrm{~h}$. Complete medium was then used for culture for $24 \mathrm{~h}$, following which the cells were collected for subsequent experiments.

Reverse transcription-quantitative polymerase chain reaction (RT-qPCR) analysis. Total RNA from the cells and clinical samples were obtained using the RNAiso Plus (Takara Bio, Inc., Otsu, Japan) and TRIzol LS reagent (Invitrogen; Thermo Fisher Scientific, Inc.) separately. A total of $0.5 \mu \mathrm{g}$ RNA from each sample was reverse transcribed into cDNA using the PrimeScript $^{\mathrm{TM}}$ RT reagent kit (Takara Bio, Inc., Otsu, Japan) according to the manufacturer's protocol. The mRNA expression levels were quantified using the standard $\mathrm{qPCR}$ protocol with SYBR Premix Ex Taq (Takara Bio, Inc.), which consisted of 40 cycles $\left(95^{\circ} \mathrm{C}\right.$ for $10 \mathrm{sec}, 60^{\circ} \mathrm{C}$ for $10 \mathrm{sec}$ and $72^{\circ} \mathrm{C}$ for $10 \mathrm{sec})$ following an initial denaturation step $\left(95^{\circ} \mathrm{C}\right.$ for $\left.2 \mathrm{~min}\right)$. Relative expression levels of transcripts were normalized to GAPDH and calculated using the $2^{-\Delta \Delta C q}$ formula as previously described $(15,16)$. The primer sequences used are listed in Table I.

Western blot assay. The VSMCs were grown in a 6-well plate. Total proteins were extracted by RIPA lysis buffer containing PMSF (Beyotime Institute of Biotechnology, Shanghai, China) at $4^{\circ} \mathrm{C}$ for $30 \mathrm{~min}$. The supernatant protein levels were determined by standard BCA assay. An equal quantity of protein $(50 \mu \mathrm{g})$ was loaded onto a $10 \%$ gel for sodium dodecyl sulfate polyacrylamide gel electrophoresis and then transferred onto a PVDF membrane (EMD Millipore, Billerica, MA, USA) following electrophoresis. Following the blocking of non-specific binding sites for $1 \mathrm{~h}$ at room temperature with $5 \%$ non-fat milk in TBST (Boster Biological Technology, Pleasanton, CA, USA), the membranes were individually incubated overnight with primary antibody anti-ROR2 (1:1,000, cat. no. 10616-1-AP; ProteinTech Group, Inc.), anti-RhoA (1:1,000, 10749-1-AP; ProteinTech Group, Inc.), anti-PCNA (1:1,000, cat. no. 10205-2-AP; ProteinTech Group, Inc.) and anti-MMP9 (1:1,000, 10375-2-AP; ProteinTech Group, Inc.) at $4^{\circ} \mathrm{C}$. Membranes were washed in PBS with $0.1 \%$ Tween-20 and incubated with horseradish peroxidase-conjugated sheep anti-mouse IgG and sheep anti-rabbit IgG secondary antibodies (1:3,000; cat. nos. ZDR-5307 and ZDR-5306; OriGene Technologies, Inc. Beijing, China) at room temperature for $2 \mathrm{~h}$, according to the manufacturer's instructions. The protein bands were revealed using the electrochemiluminescence method and images were captured using a BioSpectrum Gel Imaging system (Bio-Rad Laboratories, Inc., Hercules, CA, USA).

Cell Counting Kit-8 (CCK8) assay (4). Following appropriate treatment, the viability of the VSMCs cultured in 96-well plates was measured using the CCK8 assay kit (Dojindo Molecular Technologies, Inc., Kumamoto, Japan), as previously described.
The absorbance at $450 \mathrm{~nm}$ was measured. Three controls were set in each group and the whole experiment was repeated three times.

Wound-healing assay. The VSMCs were grown in medium to a confluence of $80-100 \%$ prior to the cell monolayer being scratched using a sterile micropipette tip. Following washes with PBS, fresh DMEM was added. Images of the wounded area were captured immediately following the scratch and $24 \mathrm{~h}$ later to monitor VSMC migration into the wounded region. The migratory abilities were quantified by measuring the total number of cells in the scratched regions.

Transwell assays. A Transwell invasion assay was performed as described previously (15). In brief, the VSMCs were loaded onto the upper well of the Transwell chamber with an $8-\mu \mathrm{m} \varphi$ pore membrane (Costar; Corning, Inc., Corning, NY, USA), precoated with Matrigel on an upper side of the chamber. The lower well was filled with $500 \mu \mathrm{l}$ of DMEM containing $10 \%$ FBS. Following incubation for $24 \mathrm{~h}$, the cells invaded to lower surface of the membrane were counted using a Leica Wild M690 operating microscope with a x40 objective (Leica Microsystems GmbH, Wetzlar, Germany).

Determination of the protein levels of active Rho. The RhoA activity assays were performed according to the manufacturer's protocol (Upstate Cell Signaling Solutions, Lake Placid, NY). Active Rho (Rho-GTP) was isolated with a GST fusion protein containing the RhoA binding domain of rhotekin (GST-rhotekin RBD). The cells were washed with ice-cold PBS and lysed on ice in cell lysis buffer. Following determining the protein concentrations using the method of Bradford, $250 \mu \mathrm{g}$ total protein per sample were incubated with GST-rhotekin RBD immobilized on glutathione-Sepharose $4 \mathrm{~B}$ beads (GE Healthcare Life Sciences) at $4^{\circ} \mathrm{C}$ for $60 \mathrm{~min}$. The beads were pelleted and washed three times with lysis buffer. Bound GTP-RhoA was eluted from the beads with SDS gel sample buffer, boiled for $5 \mathrm{~min}$ and separated by $12 \%$ SDS-PAGE. The active Rho proteins were detected by immunoblotting using anti-RhoA (ProteinTech Group, Inc.) antibody. Signals were quantified using ImageJ software $2 \mathrm{x}$ (National Institutes of Health, Bethesda, MD, USA).

Statistical analysis. All results are presented as the mean \pm standard deviation. A one-way analysis of variance followed by Tukey's or Dunnett's post hoc test was used to analyze the differences between multiple groups using SPSS 13.0 (SPSS, Inc., Chicago, IL, USA) and GraphPad Prism 5.0 (GraphPad Software, Inc., La Jolla, CA, USA). Two-sided $\mathrm{P}<0.05$ was considered to indicate a statistically significant difference.

\section{Results}

Expression of lncRNA 430945 and ROR2 is upregulated in human AS tissues. Hematoxylin and eosin staining revealed a higher number of VSMCs in human AS tissues than in control tissues (Fig. 1A). To determine differentially expressed lncRNAs and their associated mRNAs in human AS tissues, microarray analysis was performed 
Table I. Primers used for quantitative polymerase chain reaction.

Name

Primer (5'-3')

lncRNA 430945

Forward: GAGGCTGAGACGGGCGACCA

ROR2

Reverse: CGTGGGCCTCTTAGAAGCAGC

GAPDH

Forward: ATTGGGAACCGAACTATTTATG

Reverse: TCTGACAGGTGCGTGGAG

Forward: CTCAACTACATGGTCTACATGTTCCA

Reverse: CTTCCCATTCTCAGCCTTGACT

lncRNA, long non-coding RNA; ROR2, receptor tyrosine kinase-like orphan receptor 2.

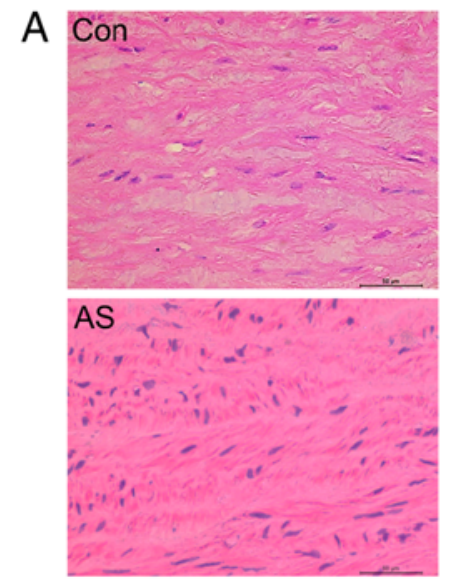

$\mathrm{B}$
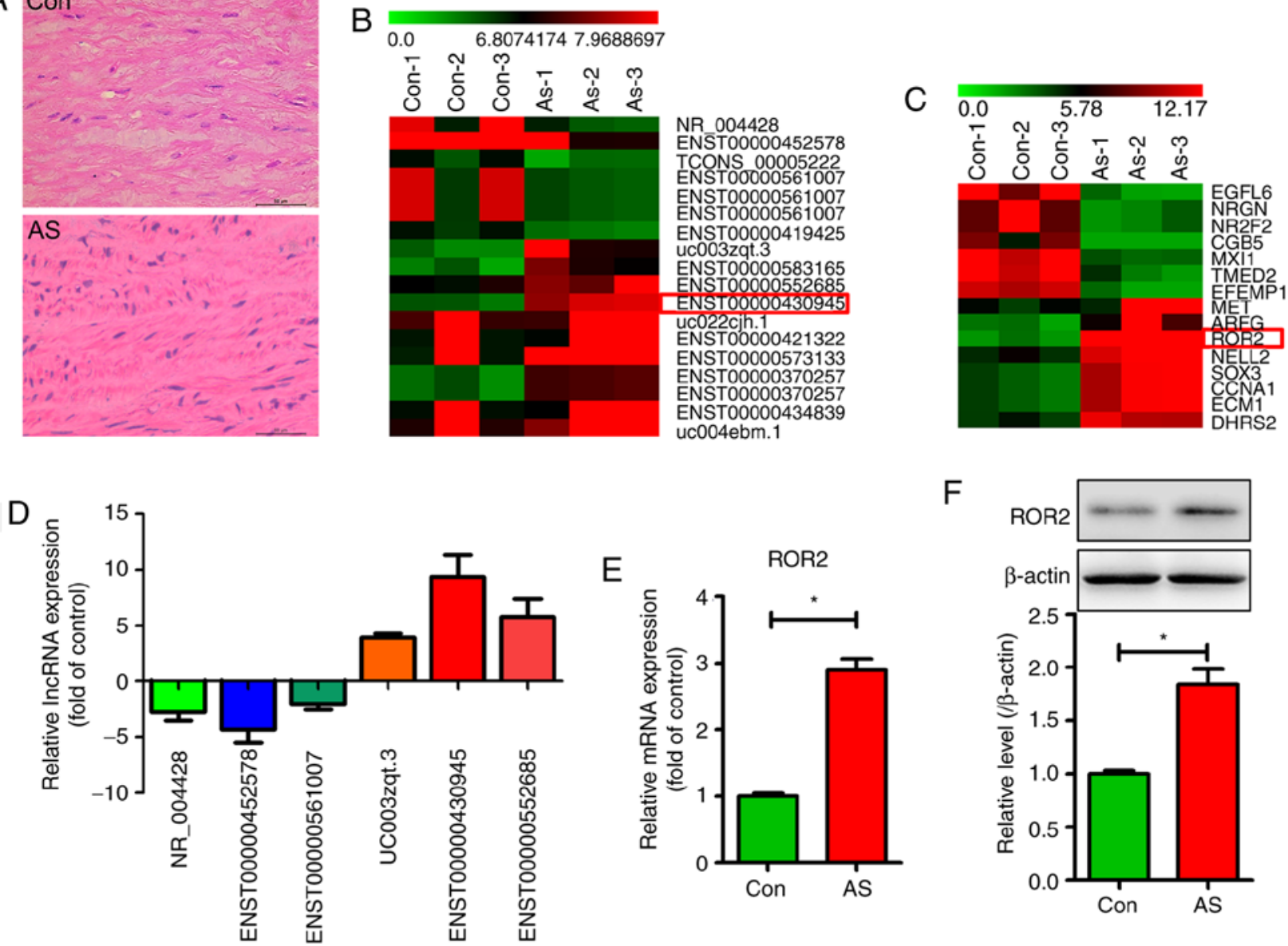

E

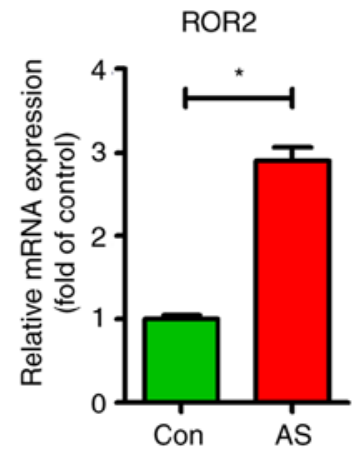

$\mathrm{F}$

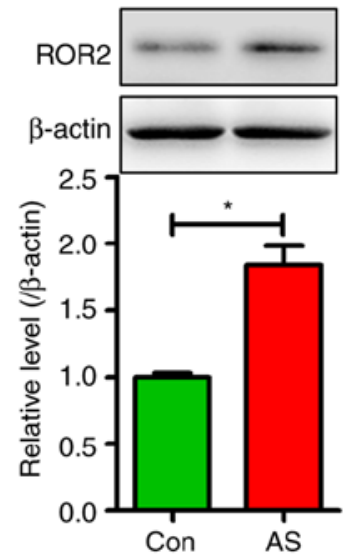

Figure 1. Expression of 1ncRNA 430945 and ROR2 are high in human AS tissues. (A) Hematoxylin and eosin-stained representative sections of aortas from human AS tissues and Con tissues. Scale bar=50 $\mu \mathrm{m}$. Subset of the differentially expressed (B) lncRNAs and (C) mRNAs detected in the AS tissues $(n=3)$ and Con tissues $(n=3)$ using microarray analysis. (D) Six lncRNAs with differential expression in AS tissues and Con tissues were validated by qPCR (E) Expression levels of ROR2 in AS tissues and Con tissues were analyzed using qPCR. * P<0.05, vs. Con ( $\mathrm{n}=3$ ). (F) Levels of ROR2 in AS tissues and Con tissues were analyzed using western blotting. The band intensities relative to $\beta$-actin are presented as the mean \pm standard deviation; $\mathrm{n}=3$; ${ }^{*} \mathrm{P}<0.05$, vs. Con. ROR2, receptor tyrosine kinase-like orphan receptor 2; lncRNAs, long non-coding RNAs; qPCR, quantitative-polymerase chain reaction; AS, atherosclerosis; Con, control (normal aortic tissues).

using atherosclerotic and normal aorta tissue samples from patients with AS and healthy donors. Differentially expressed IncRNAs were selected only when they were altered by a fold-change cut-off value of 2.0. As shown in Fig. 1B, the 20 lncRNA transcripts with the highest significant differential expression patterns are listed, with lncRNA 430945 being the most markedly upregulated. Fig. 1C shows
15 differentially expressed mRNAs identified through lncRNA microarray analysis with a fold-change cut-off of $2.0(\mathrm{P}<0.05)$, with ROR2 mRNA being the most markedly upregulated. qPCR validation indicated good reliability and reproducibility of the lncRNA 430945 change determined by RNA-seq (Fig. 1D). The qPCR and western blotting results revealed higher expression of ROR 2 at the transcriptional 
A

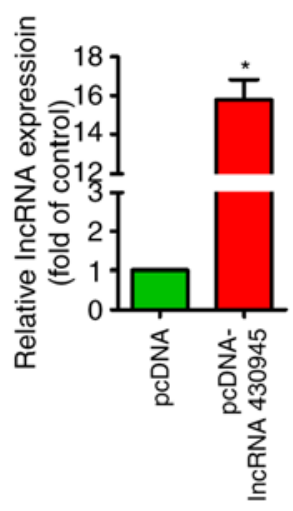

B
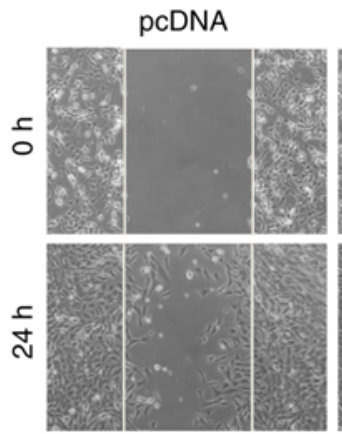

C

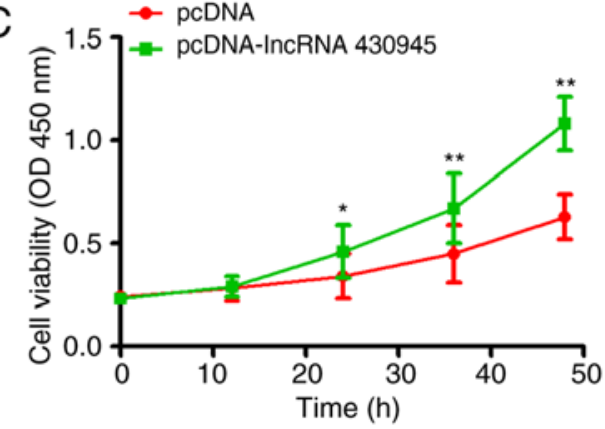

E
pcDNA-IncRNA
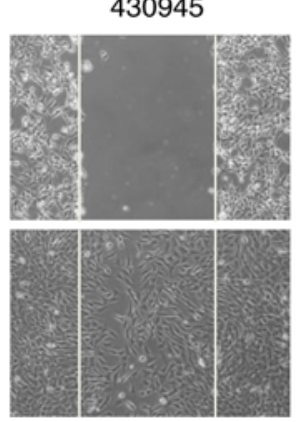

(1)
D

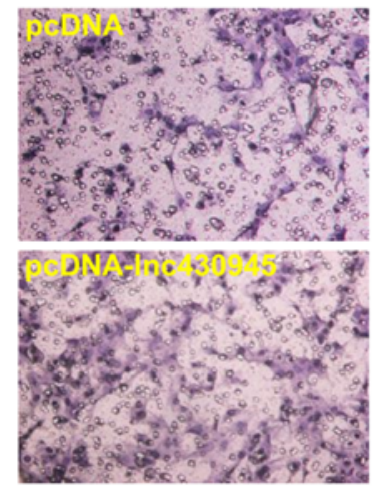

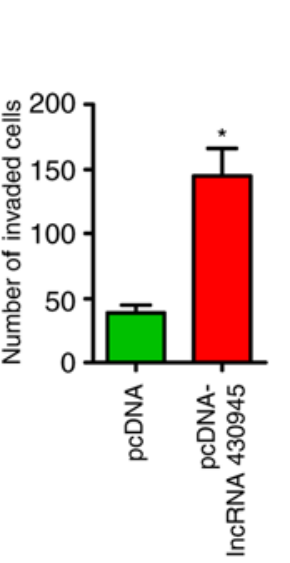

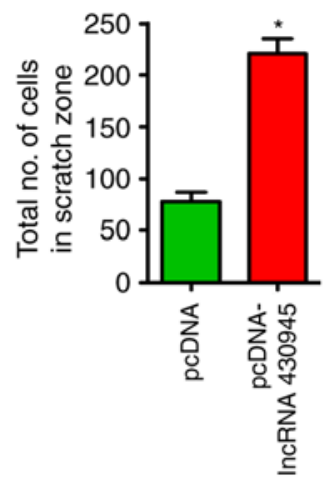

Figure 2. IncRNA 430945 promotes VSMC proliferation and migration. (A) VSMCs were transfected with pcDNA or pcDNA-lncRNA430945 for 24 h and the expression of IncRNA 430945 was analyzed by quantitative polymerase chain reaction analysis. ${ }^{*} \mathrm{P}<0.05$, vs. control ( $\mathrm{n}=3$ ). (B) VSMC monolayers were pre-treated with pcDNA or pcDNA-lncRNA430945 for $24 \mathrm{~h}$ prior to being wounded, and cells migrating to the scratch gap were quantified (magnification, $\mathrm{x} 100$ ). The data are presented as the mean \pm standard deviation from three independent experiments. ${ }^{*} \mathrm{P}<0.05$, vs. pcDNA. (C) Cell growth was assessed using a cell viability Cell Counting Kit- 8 assay following transfection with pcDNA or pcDNA-lncRNA430945 for 0, 12, 24, 36 and 48 h. ${ }^{*} \mathrm{P}<0.05$ and ${ }^{* *} \mathrm{P}<0.01$ vs. pcDNA at different times $(n=3)$. (D) Representative photomicrographs of a Transwell assay showing the quantification of VSMC invasion (magnification, $\mathrm{x} 400$ ). The number of invaded cells was measured in three separate experiments and data represent the mean \pm standard deviation ( $\mathrm{n}=3$ ). ${ }^{*} \mathrm{P}<0.05$, vs. pcDNA group. (E) VSMCs were transfected with pcDNA or pcDNA-lncRNA430945 for $24 \mathrm{~h}$ and total cell lysates were analyzed by western blotting with anti-ROR2 antibody. ${ }^{*} \mathrm{P}<0.05$, vs. pcDNA ( $\left.\mathrm{n}=3\right)$. VSMCs, vascular smooth muscle cells; ROR2, receptor tyrosine kinase-like orphan receptor 2; lncRNAs, long non-coding RNAs.

(Fig. 1E) and translational levels (Fig. 1F) in human AS tissues than in control tissues. These results suggest that lncRNA 430945 and ROR2 are involved in the phenotypic transition of VSMCs and AS tissues.

lncRNA 430945 promotes VSMC proliferation and migration. The overexpression of IncRNA 430945 in VSMCs was induced to examine its effect on cell function (Fig. 2A). CCK8, wound-healing and Transwell assays were used to examine the effect of lncRNA 430945 on the proliferation and migration of VSMCs. Consequently, it was found that the VSMCs exhibited increased proliferation (Fig. C) and migration (Fig. 2B-D).
Furthermore, western blotting revealed that the overexpression of lncRNA 430945 significantly increased the expression of ROR2 (Fig. 2E). These results suggest that lncRNA 430945 promotes VSMC proliferation and migration, thereby accelerating the development of AS.

ROR2 is involved in lncRNA 430945-mediated AngII-induced VSMC proliferation and migration. The AngII-induced dysfunction of VSMCs is crucial in the development of AS. As lncRNA 430945 exhibited high expression in AS tissues, the present study examined whether lncRNA 430945 can influence the AngII-induced proliferation and migration of 
A

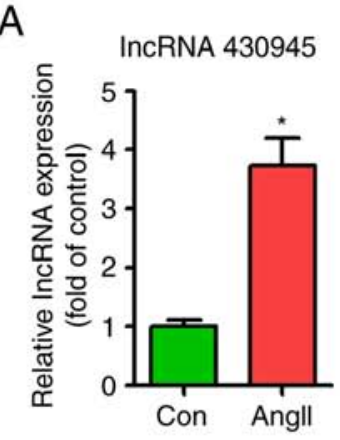

D
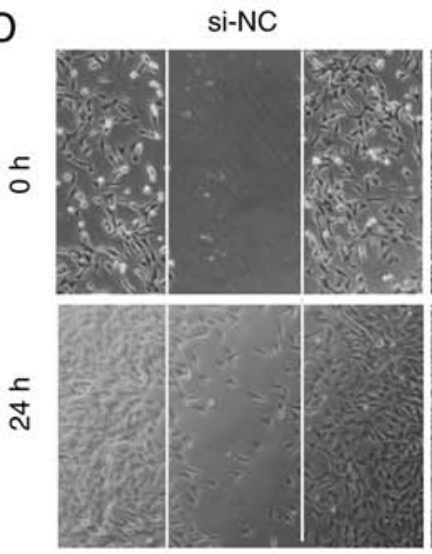

B

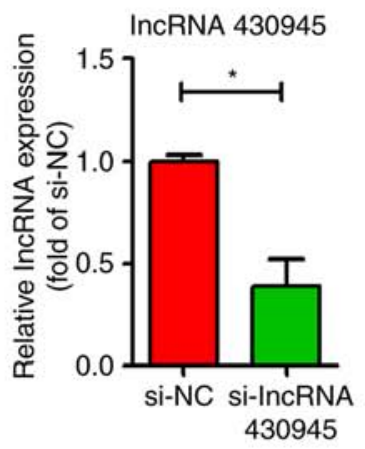

C

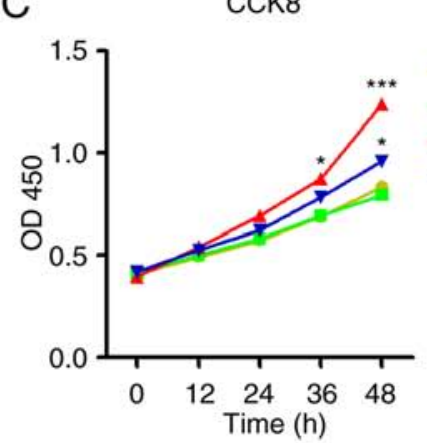

$\rightarrow$ si-NC

- si-IncRNA 430945

$\mp$ Angll

* Angll+si-IncRNA 430945

E
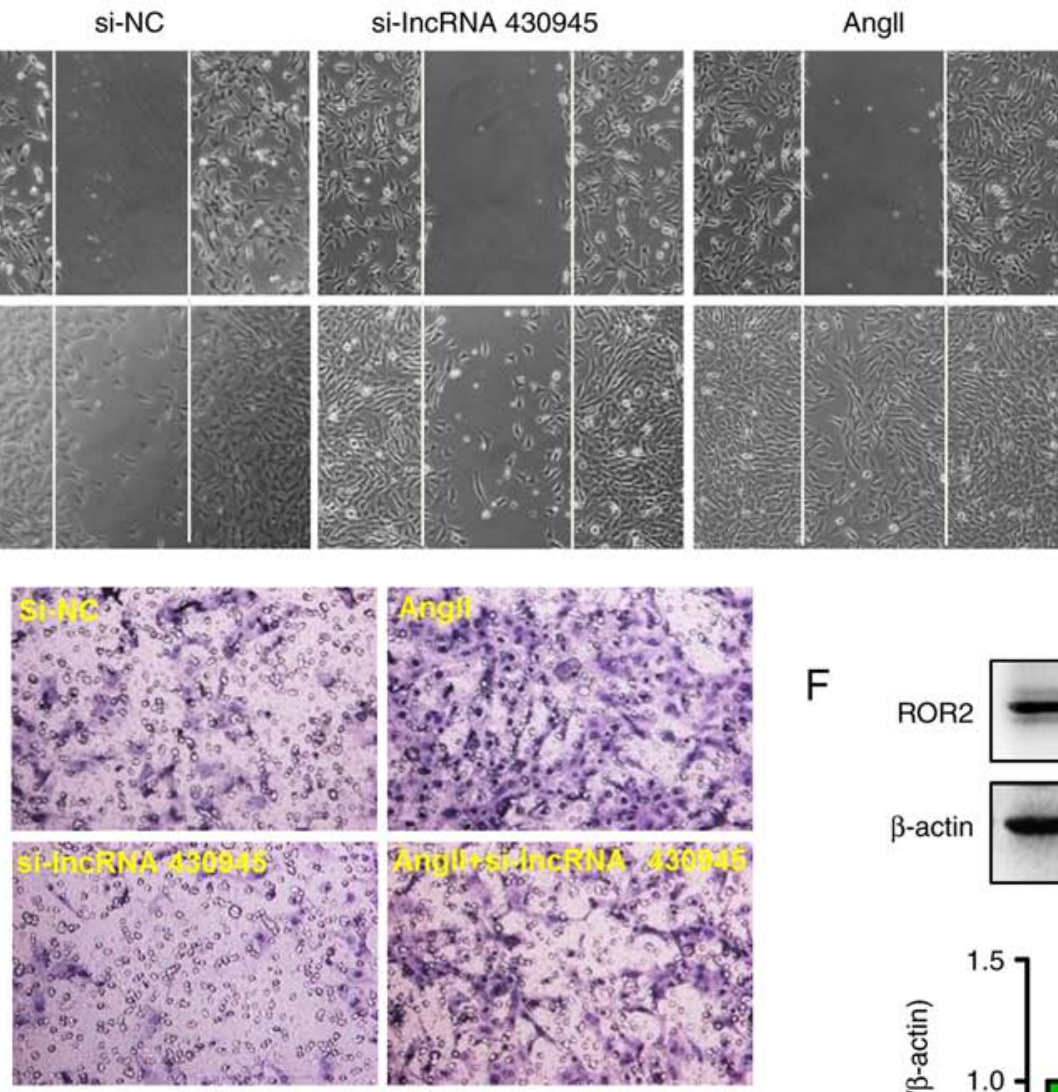

Angll+si-IncRNA
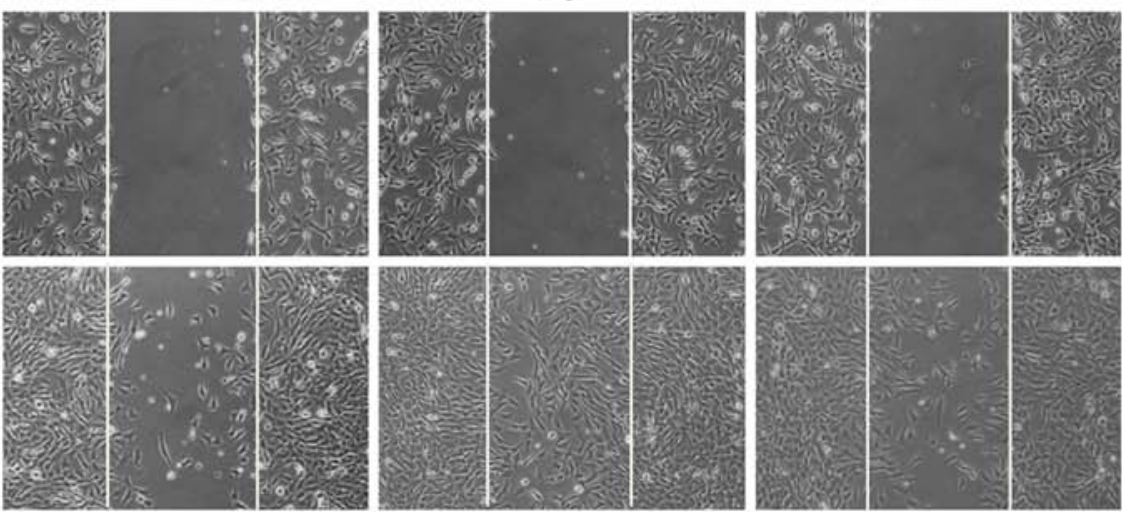

$\mathrm{F}$
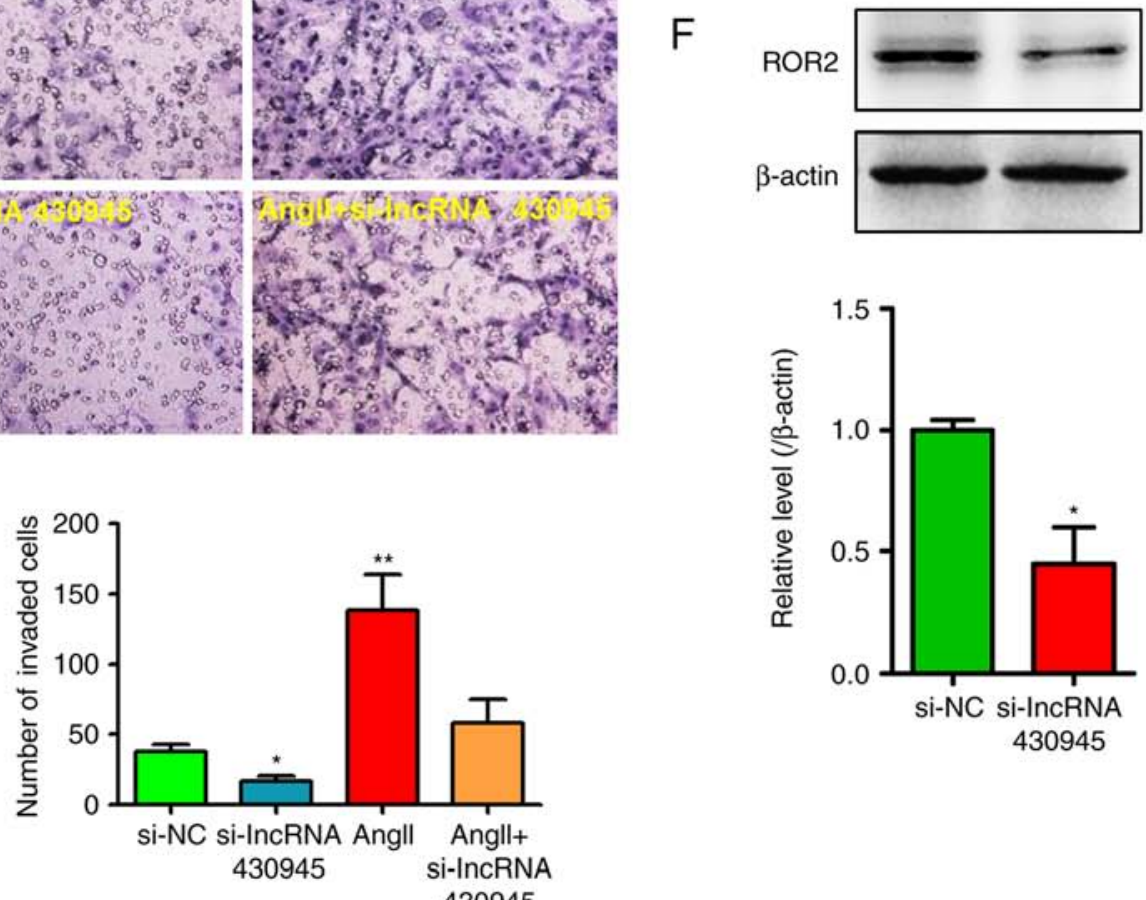

430945

Figure 3. lncRNA 430945 mediates AngII-induced VSMC proliferation, migration and invasion. (A) VSMCs were stimulated with AngII for $24 \mathrm{~h}$ and the expression of lncRNA 430945 was analyzed using qPCR. ${ }^{\mathrm{P}}<0.05$, vs. control $(\mathrm{n}=3$ ). (B) VSMCs were transfected with non-specific siRNA (si-NC) or lncRNA 430945-specifc siRNA (si-lncRNA 430945) for $24 \mathrm{~h}$ and the expression of lncRNA 430945 was analyzed using qPCR. ${ }^{*} \mathrm{P}<0.05$, vs. control (n=3). (C) VSMCs were pre-treated with si-NC or si-lncRNA 430945 for $24 \mathrm{~h}$ prior to being stimulated with AngII for an additional 0, 12, 24, 36 or 48 h. Cell growth was assessed using the cell viability Cell Counting Kit- 8 assay. ${ }^{*} \mathrm{P}<0.05$ and ${ }^{* * * *} \mathrm{P}<0.001$, vs. si-NC at different time $(\mathrm{n}=3)$. (D) VSMC monolayers were pre-treated with si-NC or si-lncRNA 430945 for $24 \mathrm{~h}$ prior to being wounded and then stimulated with or without AngII for 0 and $24 \mathrm{~h}$, and cell migration was assessed under phase-contrast light microscopy (magnification, x100). (E) Representative photomicrographs of a Transwell assay show the quantification of VSMC invasion (magnification, $\mathrm{x} 400$ ). The number of invaded cells was measured in three separate experiments and data represent the mean \pm standard deviation $(\mathrm{n}=3) .{ }^{*} \mathrm{P}<0.05$ and $^{* *} \mathrm{P}<0.01$, vs. si-NC. (F) VSMCs were transfected with si-NC or si-lncRNA 430945 for $24 \mathrm{~h}$ and total cell lysates were analyzed by western blotting with anti-ROR2 antibody. "P<0.05, vs. si-NC ( $\mathrm{n}=3)$. VSMCs, vascular smooth muscle cells; ROR2, receptor tyrosine kinase-like orphan receptor 2; lncRNAs, long non-coding RNAs; si-, small interfering RNA; NC, negative control; AngII, angiotensin II; qPCR, quantitative polymerase chain reaction. 
VSMCs. The expression of lncRNA 430945 was determined in VSMCs following AngII treatment and transfection with lncRNA 430945 siRNA for 24-48 h, following which PCR was performed. As shown in Fig. 3A and B, AngII treatment significantly increased the expression of lncRNA 430945 in VSMCs; the expression of lncRNA 430945 in VSMCs transfected with si-lncRNA 430945 was markedly lower than that in VSMCs transfected with si-NC. Furthermore, the downregulation of lncRNA 430945 in VSMCs transfected with si-lncRNA 430945 led to significantly higher suppression of AngII-induced proliferation and migration than in the VSMCs transfected with si-NC (Fig. 3C-E). The, siRNA-mediated knockdown of lncRNA 430945 reduced the AngII-induced and basal expression of ROR2 (Fig. 3F). Together, these results suggest that lncRNA 430945 affects the expression of ROR2 and thereby mediates AngII-induced VSMC proliferation and migration.

IncRNA 430945 promotes the proliferation and migration of VSMCs in a ROR2-dependent manner. As the overexpression of lncRNA 430945 was linked to the upregulation of ROR2 in VSMCs and in AS tissues, a decrease in the expression of ROR2 in VSMCs was induced using si-ROR2 to further clarify the association between lncRNA 430945 and ROR2. Western blotting showed that the expression of ROR2 in the si-ROR2-treated VSMCs was significantly lower than that in the si-NC-treated VSMCs (Fig. 4A). The CCK8 assay showed that VSMC proliferation was markedly lower following transfection with si-ROR2 than that in the si-NC-transfected VSMCs and control cells (Fig. 4B). As the upregulation of lncRNA 430945 increased cell proliferation and migration, the present study ascertained whether VSMC proliferation and migration were also modified in response to increased lncRNA 430945, and whether si-ROR2 influenced the cell proliferation and migration induced by lncRNA 430945. As shown in Fig. 4C-E, si-ROR2 significantly reduced VSMC proliferation and migration, irrespective of whether IncRNA 430945 was upregulated. These data revealed that lncRNA 430945 promotes VSMC proliferation and migration by upregulating the expression of ROR2.

LncRNA 430945 activates the RhoA signaling pathway in VSMCs. The expression of ROR 2 affects the RhoA signaling pathway, which is an essential intracellular signaling pathway, and is involved in cellular proliferation and migration during the development of AS $(17,18)$. Therefore, the present study aimed to discern whether lncRNA 430945 regulates VSMC proliferation and migration via the ROR2/RhoA signaling pathway. The results revealed that the levels of GTP-RhoA, PCNA and MMP9 in VSMCs transfected with si-lncRNA 430945 were markedly lower than those in VSMCs transfected with si-NC (Fig. 5A). By contrast, the levels of GTP-RhoA, PCNA and MMP9 in VSMCs transfected with pcDNA-lncRNA430945 were markedly higher than those in VSMCs transfected with pcDNA-NC (Fig. 5B). The total RhoA levels in all the cells remained unchanged. This indicated that lncRNA 430945 is positively correlated with the ROR2/RhoA signaling pathway in VSMCs.

The present study also attempted to elucidate the mechanisms underlying lncRNA 430945-mediated AngII-induced
VSMC proliferation and migration. Treating VSMCs with AngII significantly increased the expression of lncRNA 430945 and ROR2, and activated the GTP-RhoA signaling pathway, whereas repressing the expression of IncRNA 430945 and ROR 2 inhibited the VSMC proliferation and migration induced by AngII. In addition, a mouse model of carotid artery ligation-induced intimal thickening and proliferation was used to evaluate the protective effect of si-lncRNA 430945 on vascular remodeling. As shown in Fig. 5C and D, si-lncRNA 430945 treatment following surgery effectively inhibited vascular intimal hyperplasia compared with that in the si-NC treatment group.

\section{Discussion}

Activated VSMCs may contribute to the progression and eventual rupture of atherosclerotic plaques (19). Therefore the main purpose of the present study was to identify potential molecular targets to inhibit VSMC proliferation and migration. It was found that AngII increased lncRNA 430945-mediated VSMC proliferation and migration by upregulating the expression of ROR2 and activating the RhoA signaling pathway. By contrast, inhibiting lncRNA 430945 or ROR2 suppressed the AngII-induced VSMC proliferation and migration by inactivating the RhoA signaling pathway.

It has been shown that lncRNAs are involved in the development of AS, and certain lncRNAs may be promising as potential drug targets for treating AS $(20,21)$. LncRNAs HOXC-AS1 and HOXC6 are downregulated in carotid AS (22). In addition, Inc00113 is upregulated in AS, which indicates it may promote cell survival, proliferation and migration by activating the PI3K/Akt/mTOR signaling pathway (21). To clarify the role of lncRNAs in AS in the present study, microarray analysis and lncRNA-mRNA co-expression profiling were performed by using tissue samples from patients with AS and healthy volunteers. It was found that lncRNA 430945 and ROR2 were considerably upregulated, which were selected for ascertaining their role in AS. It has also been reported that the expression of IncRNA-RNCR3 is high in the mouse and human aortic AS. The proliferation and migration of VSMCs is suppressed when the expression of lncRNA-RNCR3 decreases, suggesting that lncRNARNCR3 is a putative target in treating AS (23). Another regulatory factor, lincRNA-p21, regulates neointima formation, VSMC proliferation, apoptosis and atherosclerotic plaque development by enhancing the activity of p53 (24), suggesting that lncRNAs may serve as therapeutic targets for treating AS and other vascular diseases. To the best of our knowledge, the present study is the first to report that a link exists between lncRNA 430945 and AS. The downregulation of lncRNA 430945 significantly suppressed VSMC proliferation and migration via ROR2, indicating that lncRNA 430945 and ROR2 are important in the development of AS.

Rho small GTPases regulate a variety of cellular functions, including proliferation, migration and invasion. RhoA is required for atherosclerotic AngII-induced VSMC proliferation. The present study also confirmed that lncRNA 430945 regulated the RhoA signaling pathway. In addition, IncRNA 430945 modulated the expression of ROR2, which in turn regulated the expression of PCNA and MMP9 in VSMCs. 
A
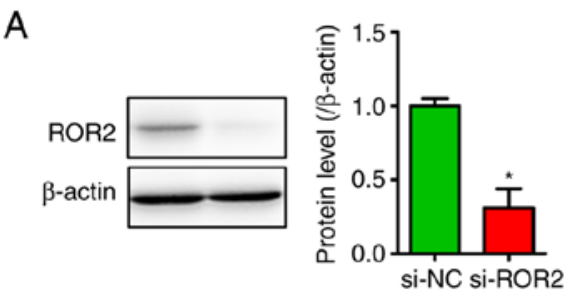
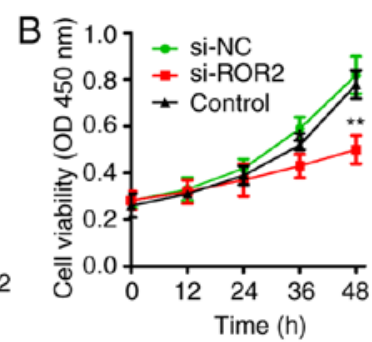

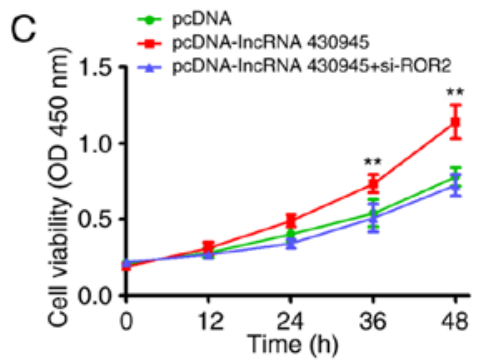

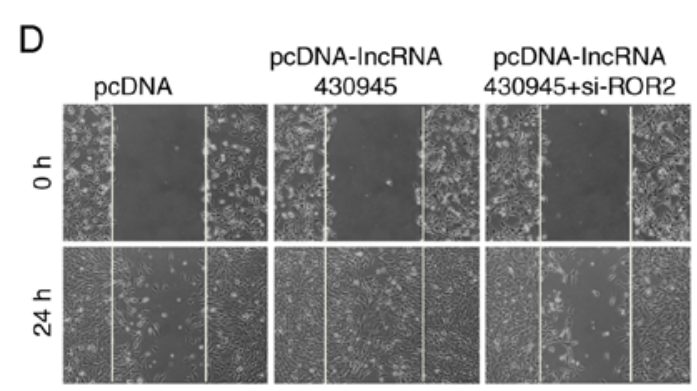
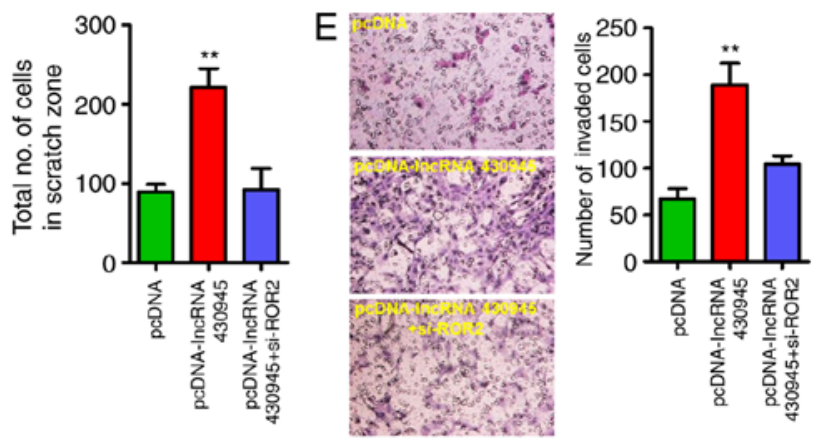

Figure 4. IncRNA 430945 promotes ROR2-dependent VSMC proliferation and migration. (A) ROR2 knockdown in VSMCs transfected with ROR2-specifc siRNA (si-ROR2) for $24 \mathrm{~h}$ was confirmed by western blotting with anti-ROR2 antibody. ${ }^{*} \mathrm{P}<0.05$, vs. si-NC ( $\mathrm{n}=3$ ). (B) Cell growth was assessed by a cell viability CCK8 assay following transfection with si-NC or si-ROR2 for $0,12,24,36$ and $48 \mathrm{~h} .{ }^{* *} \mathrm{P}<0.01$, vs. si-NC at different time (n=3). (C) VSMCs were pre-treated with si-lncRNA 430945 for $24 \mathrm{~h}$ prior to being transfected with pcDNA or pcDNA-lncRNA430945 for an additional 0, 12, 24, 36 or 48 h. Cell growth was assessed by a cell viability CCK8 assay. ${ }^{* *} \mathrm{P}<0.01$, vs. pcDNA at different time $(\mathrm{n}=3)$. (D) VSMC monolayers were pre-treated with $\mathrm{cDNA}$ or pcDNA-lncRNA430945 for $24 \mathrm{~h}$ prior to being wounded and then transfected with or without si-ROR2 for 0 and $24 \mathrm{~h}$, and cell migration was assessed under phase-contrast light microscopy (magnification, $x 100)$. Quantification of cells migrating into the scratch gap. ${ }^{* *} \mathrm{P}<0.01$, vs. pcDNA $(\mathrm{n}=3)$. (E) Transwell assay for the quantification of VSMC invasion (magnification, $\mathrm{x} 400$ ). ${ }^{* *} \mathrm{P}<0.01$ vs. pcDNA $(\mathrm{n}=3$ ). VSMCs, vascular smooth muscle cells; ROR2, receptor tyrosine kinase-like orphan receptor 2; lncRNAs, long non-coding RNAs; si-, small interfering RNA; NC, negative control; CCK8, Cell Counting Kit-8.
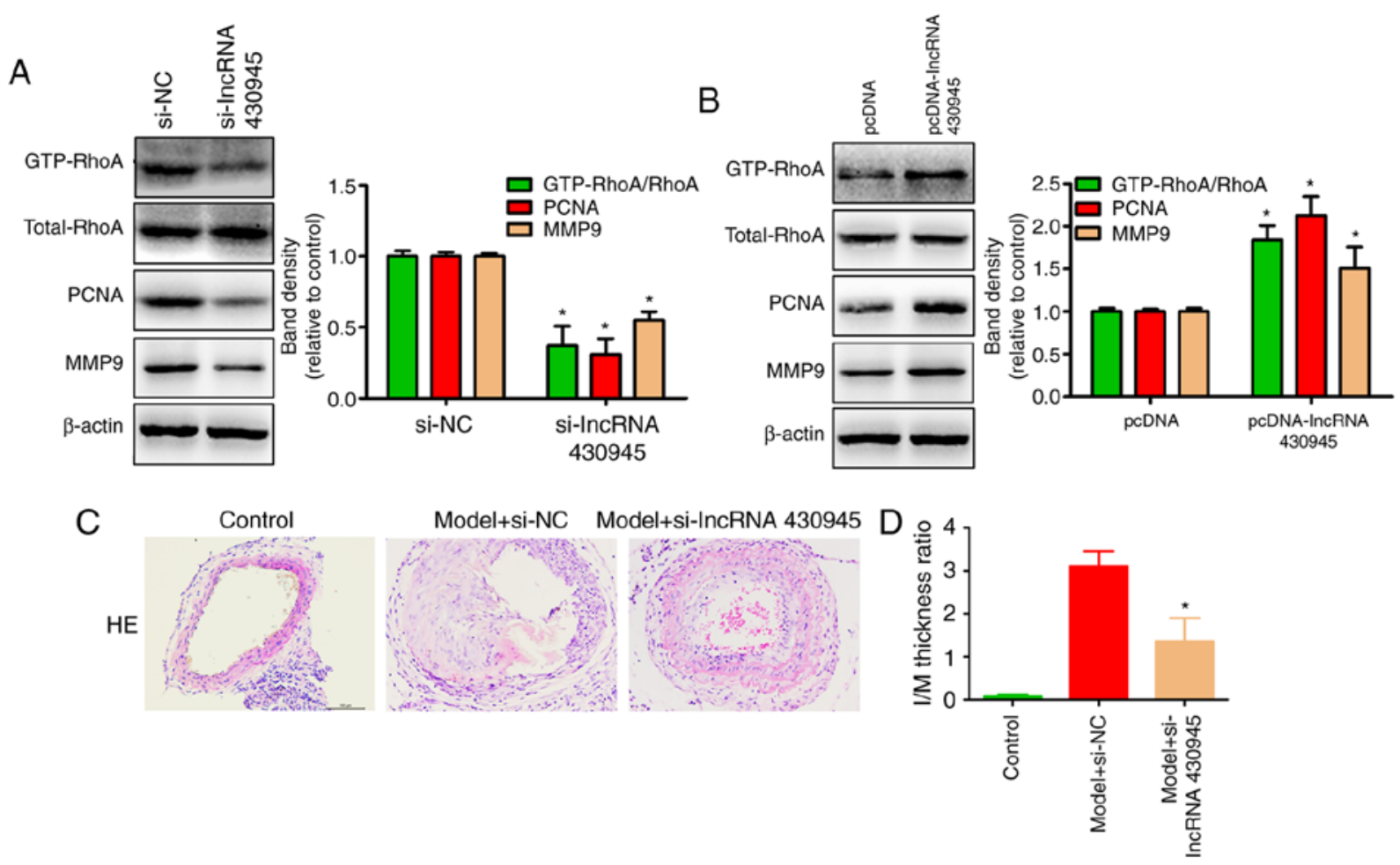

Figure 5. IncRNA 430945 activates the RhoA signaling pathway and promotes the expression of PCNA and MMP9 in VSMCs. VSMCs were transfected with (A) si-NC and si-lncRNA 430945 or with (B) pcDNA and pcDNA-lncRNA430945. GTP-bound RhoA levels were determined by GST pull-down and immunoblotting with anti-RhoA antibody. The expression levels of PCNA and MMP9 was analyzed by western blotting with anti-PCNA and anti-MMP9 antibodies. Band intensities were measured and normalized to $\beta$-actin and are shown below. ${ }^{*} \mathrm{P}<0.05$, vs. si-NC or pcDNA ( $\mathrm{n}=3$ ). (C) Representative images of HE-stained carotids of C57BL/6 mice treated with si-NC or si-lncRNA 430945 for 14 days. (D) Morphometric measurements of I/M ratio in carotids (magnification, $\mathrm{x} 200$ ). Data are presented as the mean \pm standard deviation ( $\mathrm{n}=5$ per group). $\mathrm{P}<0.001$, vs. Model + si-NC group. lncRNAs, long non-coding RNAs; si-, small interfering RNA; NC, negative control; PCNA, proliferating cell nuclear antigen; MMP9, matrix metallopeptidase 9; RhoA, Ras homolog gene family member $\mathrm{A}$; HE, hematoxylin and eosin; I/M, intima/media. 
Therefore, IncRNA 430945/ROR2 promoted VSMC proliferation and migration in a hypertensive state by activating the RhoA pathway. Resveratrol has been shown to prevent AngII-induced hypertension and SMC proliferation via AMPK activation and inhibition of the RhoA/Rho-associated protein kinase signaling pathway in vivo and in vitro, highlighting a novel therapeutic target with anti-atherosclerotic properties (25). It has also been found that naringin treatment inhibits the TNF $\alpha$-induced RhoA pathway in VSMCs, which provides a theoretical basis for the prevention of AS (26). In view of this, it is evident that lncRNA 430945 can activate the RhoA pathway, suggesting that the inactivation of IncRNA 430945 or RhoA signaling may suppress the development and progression of AS.

In conclusion, the findings reported here provide evidence that IncRNA 430945 promotes VSMC proliferation and migration by upregulating the expression of ROR2 and activating the RhoA signaling pathway. It was shown that the upregulation of IncRNA 430945 promoted ROR2 and RhoA signaling pathways, contributing to the AngII-induced proliferation and migration of VSMCs and AS. Further investigations are necessary to validate the potential of these results, so that an effective therapeutic strategy can be designed to potentially treat AS in humans.

\section{Acknowledgements}

Not applicable.

\section{Funding}

No funding was received.

\section{Availability of data and materials}

The datasets used and/or analyzed during the current study are available from the corresponding author on reasonable request.

\section{Authors' contributions}

CC, LL, YM, GZ, YS, XG, BZ, MT and HW performed the experiments, data collection and interpretation. XS was involved in the design and coordination of experiments and the acquisition of data. XW participated in the study design, data collection, analysis of data and preparation of the manuscript. $\mathrm{CC}$ participated in the study design, analysis and interpretation of data and drafting of the manuscript. All authors read and approved the final manuscript.

\section{Ethics approval and consent to participate}

The present study was approved by the Ethical Committee of Tangshan Workers Hospital, Hebei Medical University. Experimental procedures were implemented in accordance with to the guidelines and regulations of Hebei Medical University and written informed consent was obtained from all patients.

\section{Patient consent for publication}

Not applicable.

\section{Competing interests}

The authors declare that they have no competing interests.

\section{References}

1. Lusis AJ: Atherosclerosis. Nature 407: 233-241, 2000.

2. Heeneman S, Sluimer JC and Daemen MJ: Angiotensin-converting enzyme and vascular remodeling. Circ Res 101: 441-454, 2007.

3. Yang L, Froberg JE and Lee JT: Long noncoding RNAs: Fresh perspectives into the RNA world. Trends Biochem Sci 39: 35-43, 2014.

4. Lu QB, Wang HP, Tang ZH, Cheng H, Du Q, Wang YB, Feng WB, Li KX, Cai WW, Qiu LY and Sun HJ: Nesfatin-1 functions as a switch for phenotype transformation and proliferation of VSMCs in hypertensive vascular remodeling. Biochim Biophys Acta-Mol Basis Dis 1864: 2154-2168, 2018.

5. Li H, Liu X, Zhang L and Li X: LncRNA BANCR facilitates vascular smooth muscle cell proliferation and migration through JNK pathway. Oncotarget 8: 114568-114575, 2017.

6. Rinn JL and Chang HY: Genome regulation by long noncoding RNAs. Annu Rev Biochem 81: 145-166, 2012.

7. Ballantyne MD, Pinel K, Dakin R, Vesey AT, Diver L, Mackenzie R, Garcia R, Welsh P, Sattar N, Hamilton G, et al: Smooth muscle enriched long noncoding RNA (SMILR) regulates cell proliferation. Circulation 133: 2050-2065, 2016.

8. Onishi I, Suzuki H, Onishi N, Takada R, Kani S, Ohkawara B, Koshida I, Suzuki K, Yamada G, Schwabe GC, et al: The receptor tyrosine kinase Ror2 is involved in non-canonical Wnt5a/JNK signalling pathway. Genes Cells 8: 645-654, 2003.

9. He F, Xiong W, Yu X, Espinoza-Lewis R, Liu C, Gu S, Nishita M, Suzuki K, Yamada G, Minami Y and Chen Y: Wnt5a regulates directional cell migration and cell proliferation via Ror2-mediated noncanonical pathway in mammalian palate development. Development 135: 3871-3879, 2008.

10. Hung CN, Huang HP, Lii CK, Liu KL and Wang CJ: Sulforaphane inhibits smooth muscle cell proliferation and migration by reducing MMP-9 activity via the Ras and RhoA/ROCK pathways. J Funct Foods 5: 1097-1107, 2013.

11. Cario-Toumaniantz C, Ferland-Mccollough D, Chadeuf G, Toumaniantz G, Rodriguez M, Galizzi JP, Lockhart B, Bril A, Scalbert E, Loirand G and Pacaud P: RhoA guanine exchange factor expression profile in arteries: Evidence for a Rho kinase-dependent negative feedback in angiotensin II-dependent hypertension. Am J Physiol-cell Physiol 302: C1394-C1404, 2012.

12. Shi Y, Fu Y, Tong W, Geng Y, Lui PP, Tang T, Zhang X and Dai K: Uniaxial mechanical tension promoted osteogenic differentiation of rat tendon-derived stem cells (rTDSCs) via the Wnt5a-RhoA pathway. J Cell Biochem 113: 3133-3142, 2012.

13. Zhou T, Ding JW, Wang XA and Zheng XX: Long noncoding RNAs and atherosclerosis. Atherosclerosis 248: 51-61, 2016.

14. Allagnat F, Haefliger JA, Lambelet M, Longchamp A, Bérard X, Mazzolai L, Corpataux JM and Déglise S: Nitric oxide deficit drives intimal hyperplasia in mouse models of hypertension. Eur J Vasc Endovasc Surg 51: 733-742, 2016.

15. Ma D, Zheng B, Suzuki T, Zhang R, Jiang C, Bai D, Yin W, Yang Z, Zhang X, Hou L, et al: Inhibition of KLF5-Myo9b-RhoA pathway-mediated podosome formation in macrophages ameliorates abdominal aortic aneurysmnovelty and significance. Circ Res 120: 799-815, 2017.

16. Livak KJ and Schmittgen TD: Analysis of relative gene expression data using real-time quantitative PCR and the 2(-Delta Delta C(T)) method. Methods 25: 402-408, 2001.

17. Zhang CJ, Liu Z, Shi Z, Liao DF and Li Qin L: Wnt5a/Ror2 pathway contributes to the regulation of cholesterol homeostasis and inflammation response in atherosclerosis. Atherosclerosis 32: 64-64, 2018.

18. Kiian I and Dumler I: W01.42 Rosuvastatin regulates RhoA and UPA/uPAR systems required for migration of human vascular smooth muscle cells. Atherosclerosis 5: 10, 2004.

19. Gorenne I, Kumar S, Gray K, Figg N, Yu H, Mercer J and Bennett M: Vascular smooth muscle cell sirtuin 1 protects against DNA damage and inhibits atherosclerosis clinical perspective. Circulation 127: 386-396, 2013.

20. Meng F, Yan J, Ma Q, Jiao Y, Han L, Xu J, Yang F and Liu J: Expression status and clinical significance of lncRNA APPAT in the progression of atherosclerosis. Peerj 6: e4246, 2018. 
21. Yao X, Yan C, Zhang L Li Y and Wan Q: LncRNA ENST00113 promotes proliferation, survival, and migration by activating $\mathrm{PI} 3 \mathrm{~K} / \mathrm{Akt} / \mathrm{mTOR}$ signaling pathway in atherosclerosis. Medicine (Baltimore) 97: e0473, 2018.

22. Huang C, Hu YW, Zhao JJ, Ma X, Zhang Y, Guo FX, Kang CM Lu JB, Xiu JC, Sha YH, et al: Long noncoding RNA HOXC-AS1 suppresses Ox-LDL-Induced cholesterol accumulation through promoting HOXC6 expression in THP-1 macrophages. DNA Cell Biol 35: 722-729, 2016.

23. Shan K, Jiang Q, Wang XQ, Wang YN, Yang H, Yao MD, Liu C, Li XM, Yao J, Liu B, et al: Role of long non-coding RNA-RNCR3 in atherosclerosis-related vascular dysfunction. Cell Death Dis 7: e2248, 2016.

24. Wu G, Cai J, Han Y, Chen J, Huang ZP, Chen C, Cai Y, Huang H, Yang Y, Liu Y, et al: LincRNA-p21 regulates neointima formation, vascular smooth muscle cell proliferation, apoptosis, and atherosclerosis by enhancing p53 activity. Circulation 130: $1452-1465,2014$
25. Cao X, Luo T, Luo X and Tang Z: Resveratrol prevents AngII-induced hypertension via AMPK activation and RhoA/ROCK suppression in mice. Hypertension Res 37: 803-810, 2014.

26. Lee EJ, Kim DI, Kim WJ and Moon SK: Naringin inhibits matrix metalloproteinase-9 expression and AKT phosphorylation in tumor necrosis factor-alpha-induced vascular smooth muscle cells. Mol Nutr Food Res 53: 1582-1591, 2009.

(i) (3) This work is licensed under a Creative Commons Attribution-NonCommercial-NoDerivatives 4.0 International (CC BY-NC-ND 4.0) License. 